Relations industrielles

Industrial Relations

Dénouer les conflits relationnels en milieu de travail, par Solange CormiER, Québec : Presses de l'Université du Québec, 2004, 188 p., ISBN : 2-7605-1232-0.

\title{
Luc Brunet
}

Volume 61, numéro 1, hiver 2006

URI : https://id.erudit.org/iderudit/013729ar

DOI : https://doi.org/10.7202/013729ar

Aller au sommaire du numéro

\section{Éditeur(s)}

Département des relations industrielles de l'Université Laval

\section{ISSN}

0034-379X (imprimé)

1703-8138 (numérique)

Découvrir la revue

Citer ce compte rendu

Brunet, L. (2006). Compte rendu de [Dénouer les conflits relationnels en milieu de travail, par Solange CORMIER, Québec : Presses de l'Université du Québec, 2004, 188 p., ISBN : 2-7605-1232-0.] Relations industrielles / Industrial Relations, 61(1), 185-185. https://doi.org/10.7202/013729ar

Tous droits réservés (C) Département des relations industrielles de l'Université Laval, 2006
Ce document est protégé par la loi sur le droit d'auteur. L’utilisation des services d'Érudit (y compris la reproduction) est assujettie à sa politique d'utilisation que vous pouvez consulter en ligne.

https://apropos.erudit.org/fr/usagers/politique-dutilisation/ 
Dénouer les conflits relationnels en milieu de travail, par Solange CORMIER, Québec : Presses de l'Université du Québec, 2004, 188 p., ISBN : 2-7605-1232-0.

Ce livre aborde la gestion des conflits relationnels en milieu de travail selon l'optique de la communication. Les méthodes préconisées s'inscrivent dans le courant du développement personnel continu et de l'apprentissage organisationnel. Les émotions ressenties dans un rapport d'autorité sont souvent un rappel de celles qui ont été éprouvées dans la petite enfance. Le conflit comporte ici deux niveaux, le contenu et la relation.

Ce bouquin comporte six chapitres ainsi que trois annexes comprenant des outils de diagnostic et d'intervention en gestion des conflits. Le premier chapitre porte sur la définition de la notion de conflit ainsi que sur ses composantes de type cognitif et relationnel. Le chapitre deux aborde la notion d'obstacles au dénouement des conflits relationnels. Une critique de la conception du conflit est abordée ainsi que sur les biais cognitifs. Le chapitre trois traite des étapes de la gestion des conflits en définissant dans un premier temps les étapes de l'analyse suivi des comportements en gestion des conflits (compétition, évitement, collaboration et accommodation) généralement préconisés. Ces comportements sont ensuite intégrés à l'intérieur de stratégies d'intervention. Le chapitre quatre porte plus particulièrement sur la rencontre de collaboration si l'interlocuteur a choisi cette technique pour gérer le conflit. On y retrouve ici les mécanismes relationnels et de communication sous-jacents au succès d'une telle approche. Le cinquième chapitre aborde le rôle d'une tierce partie en tant que médiateur dans la gestion d'un conflit. Les différentes étapes de la médiation sont présentées ainsi que les habiletés requises chez un individu qui veut agir dans un processus de médiation. Le chapitre six porte sur la prévention des conflits destructeurs. Le conflit est vu comme un élément de changement important quand il est bien géré, il devrait, dans cette optique, permettre de revoir les modes de fonctionnement inefficaces afin de favoriser l'innovation et la créativité. Trois annexes complètent ce livre et présentent au lecteur des outils de diagnostic portant sur l'identification de son style de gestion des conflits, une liste de réactions affectives et des critères d'analyse d'une rencontre de collaboration difficile.

Ce livre constitue un ouvrage important pour tout gestionnaire ou intervenant en milieu de travail. Les conflits étant par nature inévitable, il faut apprendre à vivre avec eux et à les harnacher pour en faire une force positive et un outil de changement fonctionnel. Cet ouvrage répond aussi à un besoin important en gestion des ressources humaines car la plupart des conflits qui surgissent en milieu de travail sont surtout d'ordre relationnel plutôt que syndical et ces conflits ne se résolvent pas par une structure juridique ou légale. Une critique que je pourrais soulever à propos de ce livre est la conception de l'auteure stipulant que la recherche de l'origine du conflit contribue peu à la compréhension de la dynamique conflictuelle présente et encore moins à l'élaboration de pistes de dénouement. La plupart des recherches en gestion des conflits mettent en lumière que la façon dont un conflit est réglé sert de point de départ au prochain, il devient important de retracer l'origine du conflit. L'étude des conflits sociétaux à travers l'histoire est assez éloquente à ce niveau.

LUC BRUNET

Université de Montréal 